\title{
CJNS Exchanges with Journals of Latin America, China and Eastern Europe
}

\author{
Harvey B. Sarnat \\ Associate Editor
}

For several years, the Canadian Journal of Neurological Sciences (CJNS) has maintained a complimentary "subscription exchange" with neurological journals of certain other countries, particularly in Latin America, China, and Eastern Europe. This arrangement increases our circulation to countries where the CJNS might not otherwise be as well known and gives us an opportunity to better appreciate work not found in the more familiar journals of North America and Western Europe. The agreement provides the same advantages to the journals with which we exchange.

We would like to share some of the papers published in these lesser known journals with our readers here in Canada. A few abstracts have therefore been selected from these exchange publications, from articles published within the past couple of years. The abstracts are translated and reproduced here for your interest. A brief description of these journals is first presented.

Neurología en Colombia and Revista Neurológica Argentina are two South American clinical neurological journals published respectively in Bogotá since 1977 and in Buenos Aires since 1975. Four issues appear annually. Review articles and case reports comprise the majority of articles, but original research also is published. Articles are in Spanish with English and Spanish abstracts of each. Some reviews by prominent North American authorities are published in Neurologia en Colombia as submitted, in English. This would defeat one purpose of this journal in bringing current information to its ordinary readers in the language they best understand.

Formal scientific studies are also published in these journals, but most Latin American medical investigators publish their most serious and original work in North American or European journals. While this tradition accomplishes the purpose of bringing greater international recognition to the authors, it also has an inherent self-destructive quality in stifling the growth and development of their own national journals by severely limiting the number and quality of submitted manuscripts. There is a sad note in the failure of such journals to receive more support from the readers who also are their principal authors. One bright note of optimism is that the editors, particularly of the Colombian journal, already recognize a potential niche for their journals that is more important for Latin America than for North America or Western Europe, the field of tropical medicine. Several original and useful contributions in parasitology have already appeared in these journals. It is this type of article that will help distinguish them from neurological publications elsewhere.

Neurological investigation is becoming increasingly more active in China. Although we know of no medical journal devoted exclusively to the clinical neurosciences that is published in the People's Republic of China, the CJNS maintains an exchange with two general Chinese medical journals published in Beijing. The Acta Physiologica Sinica and the Chinese Medical Journal each contain a large proportion of articles on neurological or neurosurgical topics. Acta Physiologica Sinica, formerly the Chinese Journal of Physiology, has had continuous publication since 1948. Articles are in Cantonese, but each is followed by an English abstract. Although fundamentally a journal of experimental physiology, as its title implies, most studies have a strong clinical orientation in addressing problems relevant to patient care. Mechanisms of anaesthesia and analgesia, including traditional Chinese herbal medicine and acupuncture, are favourite topics. The literature cited in articles includes Chinese, Soviet, and Western references.

The Chinese Medical Journal, the official publication of the Chinese Medical Association, simultaneously publishes Chinese and English language versions of each monthly issue. Each volume represents one year; the current volume number is 99 . It is mainly a clinical journal of prospective studies. case reports, and some experimental animal research similar to Western clinical journals. A section at the end of each issue is devoted to abstracts of articles recently appearing in other Chinese medical literature and book reviews. The only advertisements are for subscriptions to other Chinese publications, not all of which are medical: China Sports (in English). Chinese Literature (in French), China's Foreign Trade (in Chinese, French, and Spanish), Women of China (in English).

In Eastern Europe there are several journals almost unknown in North America. The University of Zagreb, Yugoslavia, has published Neurologija since 1952. Half of the articles are in English and half are in the Serbo-Croatian language, with bilingual abstracts provided for all. The format of the journal is familiar to the Western reader. Articles are illustrated by photomicrographs, EEG and EMG tracings, tables and graphs. Eastern European and Western medical literature are equally cited. Book reviews and notes regarding future scientific meetings conclude each issue. A few pages of advertising of medical textbooks and neurological drugs are also included. There are four numbers per annual volume, but they are combined in pairs so that actually two issues a year of approximately 75 pages each are distributed. This journal is one of the most respected medical publications in the Eastern European countries and has a long tradition of academic excellence. The CJNS began an exchange with Neurologija this year.

The Bulletin of Experimental Biology is a journal of basic medical sciences published in Moscow since 1936 by the Academy of Medical Sciences of the USSR. Articles are all in 
Russian with supplementary English abstracts. Although the journal is general in scope, about 25 percent of articles deal with topics of primary interest to neuroscientists, and many have clinical relevance. Twelve numbers a year are issued, each about 125 pages in length. There is no commercial advertising. Each issue is divided into several sections identified as Physiology, Pathological Physiology and General Pathology, Biochemistry and Biophysics, Pharmacology, Microbiology and Immunology, Oncology, Experimental Biology, Morphology and Pathomorphology, and Methods. Most articles are brief, 2 to 4 pages in length, and include tables, graphs, and illustrations. The quality of the paper is poor by comparison with most Western medical journals, but pages of good quality glossy paper frequently are inserted within articles to improve the reproduction of photomicrographs. Scanning the reference list at the end of articles gives one the impression that there are two distinct groups of Soviet scientific authors: those who cite only previous Russian work and those who are well read in the Western medical literature as well. Lest we be too critical, how many of the articles we publish cite research published in Soviet journals? More scientific communication would benefit us all.

Treatment of Cysticercosis with Praziquantil.

Gómez González J, Bustamante Zuleta E. Sánchez Pérez E. Neurología en Colombia (Bogotá. Colombia) 1985: 9: 29-35

Fifty cases of intracranial cysticercosis were treated at the National Institute of Colombia between 1980 and 1984. Dosage was standardized at $50 \mathrm{mg} / \mathrm{kg} / \mathrm{day}$ divided into six hourly increments, for 15 days, using the drug praziquantil. Symptoms, including headaches. seizures, focal deficits. mental changes, and intracranial hypertension. improved in 92\% of the patients, including some patients with severe cerebral dysfunction. Four patients treated initially with low doses required further treatment after symptoms recurred. Complications of the drug included profuse sweating in the first few days of treatment and reversible cardiac arrhythmias. A striking difference was noted in the mortality from cerebral cysticercosis in Bogotá, $80 \%$ in 40 cases reported in 1971 before praziquantil was available contrasting with no deaths in our 50 treated cases.

2.

Paracoccidiomycosis Granuloma of the Cerebellum.

Díaz D and Gómez G. Neurología en Colombia (Bogotá, Colombia) 1984; 8: 150-152

A 33-year-old farmer with a granuloma of the cerebellum is presented. The diagnosis of a mass lesion was made by CT scan, and the lesion was surgically removed followed by treatment with ketokonazole, 200-400 $\mathrm{mg} / \mathrm{day}$. This drug is preferred to amphotericin B and sulpha drugs for this type of fungal infection. The patient has remained asymptomatic a year after treatment. Eleven other cases of this intracerebral fungal granuloma are found in the literature, generally with a very poor prognosis.

3.

Evaluation of the Effect of a Combination of Gangliosides with Vitamin B Complexes in Patients with Polyneuropathies and Radiculopathies.
Muchnik S, Parola A, Traña C, Mazia C, Ferraro R and Álvarez A. Rev Neurol Arg (Buenos Aires. Argentina) 1985: 11:23-26

Clinical and electromyographic studies were performed in 26 patients with diagnoses of diabetic and alcholic polyneuropathies and radiculopathies, before and after administration of gangliosides and vitamins $B_{1}, B_{6}$, and $B_{12}$. Clinical improvement was reported in all patients. Motor nerve conduction velocities performed in median nerve also improved from $39.6 \pm 4.2 \mathrm{~m} / \mathrm{sec}$ to $45.4 \pm 4.7 \mathrm{~m} / \mathrm{sec}$ and in the deep peroneal nerve from $42.0 \pm 2.3$ to $48.1 \pm 3.6 \mathrm{~m} / \mathrm{sec}$. The EMG disclosed an increment of polyphasic motor unit potentials by the end of the treatment period of 25 days.

4.

Hereditary Paroxysmal Vertigo and Ataxia Responding to Acetazolamide.

Micheli F, de Arbelaiz R, Casas Parera I, Fernández Pardal M. Paradiso G and Matsubara P. Rev Neurol Arg (Buenos Aires. Argentina) 1984: 10: $225-230$

Six members of three generations in a family, representing both sexes, were affected by an autosomal dominant form of paroxysmal vertigo and ataxia. The average frequency of attacks was 3 per week. beginning at about 6 years and continuing into adult life. Conciousness was not altered by these attacks. Neurological examination between attacks was normal. Acetazolamide in a dose of $250-500 \mathrm{mg} /$ day in adults completely controlled the paroxysmal episodes.

5.

Classical Migraine and Prolactin.

Leston J, Guitelman A, Baigorri A, Rubin Z. Fideleff H. Espindola B and Herskovits E. Rev Neurol Arg (Buenos Aires. Argentina) 1984; 10: $211-216$

Although estrogens and progesterone have been extensively studied in migraine, prolactin has received less attention. Variations in plasma levels of prolactin were measured during a 24 day period in the ovulatory and luteal phase of 4 women with classical migraine and in 2 additional postmenopausal patients. No significant abnormal values were recorded. Plasma levels up to $25 \mathrm{ng} / \mathrm{ml}$ were measured, with some higher values related to sleep and to the postmenopausal state associated with other hormonal changes.

6.

Analgesic Effect of Electroacupuncture and Morphine Antagonized by Microinjection of Calcium Ions into Habenula or Periaqueductal Grey of the Rabbit.

Zhong-Fu Z, Bao-E K. Guo-Xi X and Ji-Sheng H. Acta Physiol Sinica (Beijing, People's Republic of China) 1985; 37: 463-470

Rabbits equipped with bilateral intracranial cannulae directed to habenula or periaqueductal grey (PAG) were given microinjections of $\mathrm{CaCl}_{2}$ solution at a dose of $15-20 \mathrm{nmol} / \mathrm{uL} / \mathrm{site}$ to be completed within 8 minutes. Pain threshhold was measured by the latency of avoidance response (head jerk) elicited by radiant heat exposure before and after intracerebral injection. Microinjection of $\mathrm{CaCl}_{2}$ produced no significant effect on the basal pain threshhold. However, the analgesic effect elicited by electroacupuncture (EA) or morphine ( $2 \mathrm{mg} / \mathrm{kg}$. intravenously) was found to be significantly reduced by more than $50 \%$ in rabbits given $\mathrm{CaCl}_{2}$ to bilateral PAG or habenular nuclei. No significant effect was 
shown when the same dose of $\mathrm{CaCl}_{2}$ was administered into the vicinity of the nuclei. implying a high specificity for the site of action. Injection of $\mathrm{CaCl}_{2}$ into bilateral habenulae was more effective than unilateral injection, although in PAG area unilateral injection was sufficient to produce a maximal effect. Intra-PAG injection of $\mathrm{CaCl}_{2}$ was significantly more effective in blocking EA analgesic ( $-86 \%)$ than in blocking morphine analgesia $(-54 \%)$. The results are consistent with the hypothesis that the ability of morphine and endogenously released opioid peptides in blocking neuronal $\mathrm{Ca}^{++}$influx in certain CNS structures including PAG and habenula may constitute the basic mechanism underlying morphine analgesia and EA analgesia.

\section{7.}

Synaptic Connections Between Monoaminergic Terminals and Intercostal Respiratory Motoneurons in Cats.

Zhi-Hua J and Shen E. Acta Physiol Sinica (Beijing, People's Republic of China) 1985: 37: 479-485

In four cats, the cut end of the internal or external intercostal nerve branches were treated with retrograde fluorescent dye propidium iodide (P1) to label the intercostal respiratory motoneurons. The fluorescence of monaminergic terminals in the PI labelled neuronal pool was also shown after processing with the method of Falck and Hillarp. Both yellowish 5-HT and greenish NA varicosities could be seen in close proximity to the dendrites or soma of the labelled respiratory motoneurons. In two other cats, terminals containing dense core vesicles formed synapses with the soma or dendrites of the HRP labelled intercostal respiratory motoneurons, as visualized in the electron microscope.

8.

\section{Tumours of the Lateral Ventricle of Brain.}

Dao-kun L. Chinese Medical Journal (Beijing, People's Republic of China) $1985 ; 98: 675-680$

Thirty patients with tumours of the lateral ventricle were operated upon in this hospital in a period of ten years, with the tumours totally removed in 24 and subtotally resected in 6 . No operative death occurred. Postoperative deep X-ray irradiation and chemotherapy were given to 17 cases. All 30 cases were followed for 1 to 9 years: 19 of them were still surviving. Of these, 15 had resumed their work while the other four were dependent. The essential points in diagnosis, treatment, as well as method of improving surgical results are discussed.

9.

Clinical and Electrophysiological Study of a Case of Isaac's Syndrome.

Xiao-fu T and Bing-xian Y. Chinese Medical Journal (Beijing, People's Republic of China) 1985; 98: 695-697

A case of Isaac's syndrome with continuous muscle fibre activity or neuromyotonia is reported. The age of onset was 10 years. The course was progressive. Muscle stiffness was the prominent feature, especially of the hands and feet. The electromyogram at rest and during sleep, electroencephalogram, motor nerve conduction velocity and nerve blocks with procaine at motor points and nerve trunk were studied. Phenytoin sodium appeared therapeutically effective. Our observations tend to support the view that the condition is an anomaly of the distal part of the motor nerve fibres. The relationship between this syndrome and "flu" with fever requires further study.
10.

Agarose-Gel Electrophoresis of the Cerebrospinal Fluid in the Diagnosis of Subacute Sclerosing Panencephalitis.

Vranješević D. Navra D and Radosavlijević B. Neurologija (Zagreb. Yugoslavia) 1984: 32: 53-60

The presence of oligoclonal bands in the cerebrospinal fluid (CSF) is described in subacute sclerosing panencephalitis (SSPE) and shows the existence of the synthesis of $\operatorname{lgG}$ within the central nervous system. By means of agarose-gel electrophoresis. we investigated 25 specimens of CSF and serum from 6 patients with SSPE. Oligoclonal banding was found in all CSF samples. This positive finding did not correlate with the stage of the illness during which the specimen was collected, and in some cases it was the first indicator of the disease. Other CSF indices also showing intrathecal synthesis of IgG also were positive. The presence of oligoclonal bands in all stages of the illness makes agarosegel electrophoresis of CSF a useful procedure in the diagnosis of SSPE. particularly in early stages.

11.

Long-Term Follow-Up of Children with Infantile Hydrocephalus.

Franulović J and Križ M. Neurologija (Zagreb. Yugoslavia) 1984: 32: $79-88$

A longitudinal study was carried out in 34 children with infantile hydrocephalus in which shunt operations were performed. Total mortality rate in the series was $14.7 \%$ during the period of observation. The intellectual development was followed during a period of 1 to 5 years. and the final I.Q. was practically unchanged with the exception of a small group ( 5 children) in whom a slight decrease was noted. The results of this prospective study were as follows: $28.5 \%$ had normal development: $38 \%$ of children showed a moderate retardation: while the remaining $33 \%$ had severely impaired neurodevelopmental function (I.Q. below 50. cerebral palsy. etc.) The thickness of the cerebral tissue measured at the time of the shunt operation seems to be a relatively useful predictive factor; children with a cerebral mantle of more than $20 \mathrm{~mm}$ had statistically significant higher intellectual performance. compared with the group of patients in whom the thickness of the brain matter was less than $20 \mathrm{~mm}$. Finally. the late complications of shunt procedures are discussed (obstruction of the Pudenz system, infections). In half of children $(51.2 \%)$ an operative revision was needed.

12.

Examination of Adenosine Cyclic 3, 5'-Monophosphate Relation and Antiepileptic Concentration in a Group of Resistant Epileptic Patients.

Trbojević-Čepe M, Hajnšek F. Stavljenić A, Arambašin M and Knežević S. Neurolgija (Zagreb. Yugoslavia) 1984; 32: 33-44

The relation of adenosine 3,5'-monophosphate (cAMP) concentration and antiepileptic drugs in a group of resistant epileptics is presented. A significant difference between the concentration of cAMP in epileptics compared with the control group has been found. No significant differences of CAMP have been observed between the control group and those with optimal of below optimal phenobarbital levels in serum. According to our results, in several treated patients the daily oscillattions of cAMP have been proved. Often these variations were followed by changes in the antiepileptic drug level in serum. 
13.

Cortical Representation of the Heart Sinus Node Zone.

Bilibin DP and Shevelev OA. Bull Exp Biol (Moscow, U.S.S.R.) 1985: 11: $517-519$

Evoked potentials (EP) in response to electrical stimulation of the heart sinus node were recorded from the cerebral cortex of cats. EP with the shortest latent period of $18-19 \mathrm{msec}$ were registered in the left and right cerebral hemispheres from cross-shaped area comprising associative field PCA and partically from primary motor and primary sensorimotor cortex. In addition, EP with a longer latency of 20-21 msec were recorded from the secondary sensorimotor cortex. EP remained unchanged after bilateral vagotomy, but disappeared after spinal cord transection at the $\mathrm{C} 2$ level. Figure 1 (below) illustrates the sites from which EP were identified.

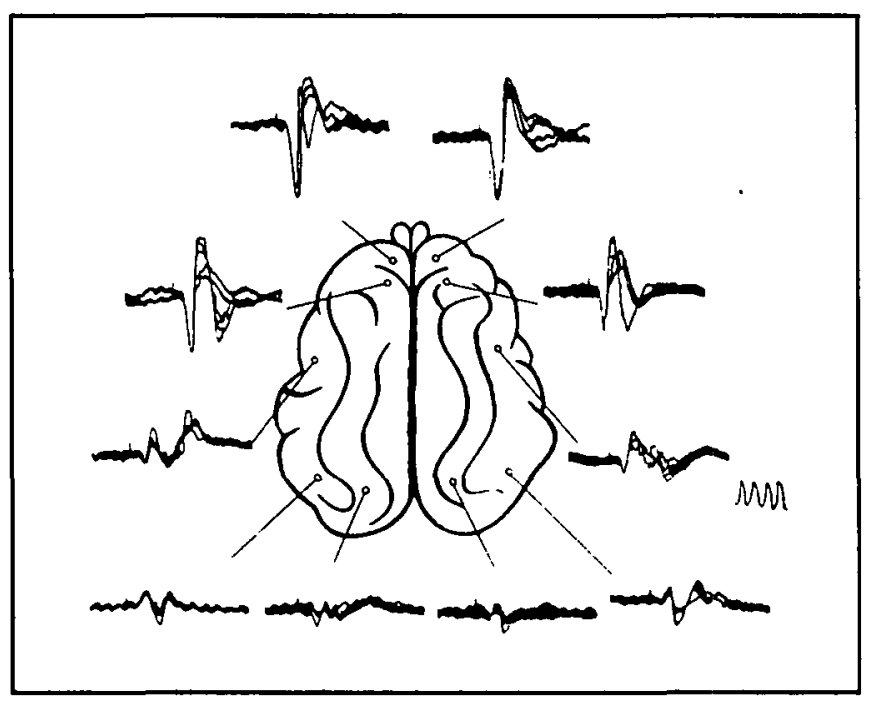

14.

Ultrastructure of the Neocortex During Rehabilitation Following Long. Term Protein-Calaric Deficiency.

Medvedev DI, Babichenko II, Eremina IZ and Kravtsova AI. Bull Exp Biol (Moscow, U.S.S.R.) 1985: 12: $751-754$

Electron microscopy of mouse neocortex was carried out during rehabilitation following long-term protein-caloric deficiency. Food rehabilitation led to partial recovery of brain ultrastructure. however a high neuronal level of secondary lysosomes and lipofuscin bodies was preserved and previous alterations in dendritic spines and synaptic contacts persisted. The addition of carnitine to the rehabilitation diet increased the number of free ribosomes in cortical neurons. A substantial development of granular endoplasmic reticulum was observed. A greater number of spine apparatus cisterns was detected, however as in conventional rehabilitation, the width of synaptic clefts and polysynaptic densities remained narrower than in control mice.

15.

Neurospecific Proteins in Malignant Brain Tumours of Man.

Berezin VA, Zhmareva EN, Brodskaya IA, Shevchenko GM, Zashko LS, Ponedilok NV, Kuznetsov AV, Kuznetsova IV and Kononova LI. Bull Exp Biol (Moscow, U.S.S.R.) 1985: 7: 68-70
The contents of neurospecific proteins S-100, GFA, and D2 were measured in malignant cerebral tumours by electrophoresis with the use of monospecific antisera. Concomitant measurements of proteins S-100 and GFA provide a more reliable diagnostic criterion of tumour histogenesis than study of each protein alone. D2 protein appeared to be the most stable specific marker.

16.

Effect of Leu-Enkephalin and Delta-Sleep-Inducing Peptide on the Release of Endogenous Noradrenaline from Rat Brain Synaptosomes.

Rozhanets VV and Anosov AK. Bull Exp Biol (Moscow. U.S.S.R.) 1985; 8: 199-201

Fluorometry was emloyed to measure the noradrenaline (NA) content in rat brain synaptosomes depending on duration of incubation. depolarization effects ( $40 \mathrm{mM} \mathrm{KCl}$ or $1.5 \mathrm{mM}$ ouabain), composition of the synaptosomal fraction and concentration of the peptides. A ten-minute incubation in a potassium medium of a suspension of light synaptosomes was used as an optimal test system for studying the peptide action. Leu-enkephalin inhibited the depolarization-induced NA release. This effect was abolished by naloxone. The delta-sleepinducing peptide (DSIP) did not influence neurotransmitter release at concentrations of $10^{-8}$ to $10^{-5} \mathrm{M}$. A mixture of amino acids imitating the amino acid composition of the DSIP influenced spontaneous release of NA. This effect is discussed in connection with the physiological action of the peptide on its intraventricular injection.

17.

The Influence of Lithium Salts on Neuropathological Syndromes of Spinal Origin.

Grafova VN and Danilova EI. Bull Exp Biol (Moscow, U.S.S.R.) 1985; 9: 273-275

Experiments on noninbred rats were performed to study the influence of lithium hydroxybutyrate on two patterns of spinal cord pathology: generalized myoclonus and a painful syndrome of spinal origin. The syndromes were induced by generators of pathologically enhanced excitation in the ventral and dorsal horns of the spinal cord. The effects of lithium chloride and sodium hydroxybutyrate were examined to compare the influence of lithium cation and hydroxybutyrate anion components. Lithium hydroxybutyrate appeared more effective, since it inhibited the generator of pathologically enhanced excitation in the appropriate structures, provoking an anticonvulsant effect in myoclonus and suppressing the painful syndrome.

18.

Influence of Hippocampal and Caudate Nucleus Destruction on Epileptic Activity During Corazol Kindling.

Kryzhanovsky GN, Shandra AA. Makulkin RF, Godlevsky LS and Moiseev IN. Bull Exp Biol (Moscow, U.S.S.R.) 1985; 10: 407-410

In randomly-bred white rats of $250-350 \mathrm{~g}$, kindling was induced by daily intra-peritoneal injections of corazol in subthreshhold (subconvulsive) doses $(30 \mathrm{mg} / \mathrm{kg}$ ). It was demonstrated that bilateral hippocampal destruction did not change the seizure threshhold, while bilateral cau- 
date ablation lowered it. Hippocampal destruction delayed the development of corazol kindling, however, and also accelerated the lowering of seizure susceptibility after corazol injections were discontinued, compared with controls. Destruction of the caudate nucleus induced more marked seizure reactions in the first 14 days after corazol injections were started. No significant differences between kindled and control animals were detected in respect of seizure severity. These data suggest an essential role of the caudate nucleus as one element in an antiepileptic system and support the concept that the hippocampus plays a role in the formation of an epileptic system underlying corazol kindling.

\section{9.}

Characteristics of Benzodiazepine Receptors in Rats with Different Predisposition to Alcoholization.

Burov YV, Yukhanonov RY and Maisky AI. Bull Exp Biol (Moscow, U.S.S.R.) 1985: 9: 311-312

A study was made of the density and affinity of benzodiazepine receptors in the cortex of the cerebral hemispheres and hippocamus of rats with differing predisposition to alcohol consumption. No differences were revealed in the parametres under study in animals with varying duration of ethanol anaesthesia or in rats after voluntary consumption of ethanol for 3.5 and 10 months. In a state of abstinence, rats with physical dependence manifested a dramatic decrease in density and affinity of benzodiazepine receptors in the cortex of the cerebral hemispheres. No changes were detected in the hippocampus.

20.

Changes in Brain Dehydrogenase and GABA-Transaminase Activity During Corazol Kindling.

Kryhanovsky GN, Moiseev IN, Shandra AA, Makulkin RF and Godlevsky LS. Bull Exp Biol (Moscow, U.S.S.R.) 1986; 1: 13-15

Experiments with $(\mathrm{CBA} \times \mathrm{C} 57 \mathrm{BL} / 6) \mathrm{F}$, mice have shown that regular corazol injections in subliminal doses stimulated seizure susceptibility (pharmacological kindling). Cytophotometric assay of the activity of oxidative metabolic enzymes (glutamate dehydrogenase, malate dehydrogenase, succinate dehydrogenase, $\alpha$-oxoglutarate dehydrogenase, lactate dehydrogenase) and GABA-transaminase in the sensorimotor cortex of kindled mice in the postconvulsive period, and 24 hours or 30 days after corazol injections were discontinued, has revealed some specific alterations of the enzymes under study that suggest the existence of two phases of energy metabolism disturbances. The first phase (24 hours after corazol injections were discontinued) is characterized by intensified succinic acid oxidation, while the second phase ( 30 days after the last injection) is characterized by anaerobic glycolysis in neuronal and glial cells. Inhibition of GABA-transaminase activity was particularly marked in the postconvulsive period. From a molecular point of view, these data may be considered to be enzymatic disturbances during stimulation of seizure susceptibility or seizure activity and, as a compensating component, ensuing anticonvulsive mechanisms and reparative processes (antagonistic principle of molecular mechanism regulation) during activation of an antiepileptic system.

21.

$\alpha$-Tocopherol Protective Effect on $\mathrm{Ca}^{++}$Transporting System of Sarcoplasmic Reticular Membranes in Hypercholesterolemia.

Kuzmina IL, Timofeev AA, Germanov SV. Levchenko LV, Kulish MA and Stoida LV. Bull Exp Biol (Moscow, U.S.S.R.) 1986: 2: 163-165

The effect of the antioxidant $\alpha$-locopherol on the calcium transporting system in sarcoplasmic reticulum (SR) of rabbit striated muscle was studied in hypercholesterolemia. $\alpha$-tocopherol administration to animals with hypercholesterolemia produced a break in the curve of temperature dependence of calcium-mediated ATPase activity at about $20^{\circ} \mathrm{C}$ that disappeared in hypercholesterolemia, increased the rate of "rapid" SH-group binding by thiol reagents, and normalized the level of unsaturated fatty acids in SR membranes without altering phospholipid content. It is suggested that the damage of $\mathrm{Ca}^{++}$-ATPase in hypercholesterolemia is mainly due to activation of lipid peroxidation.

22.

Anticonvulsant Properties of Enomelanin.

Kryzhanovsky GN, Bartsevich LB, Lobasyuk BA, Zherebin YI, Osipova LA, Mosketi KV, Braslavsky VE and Nikushkin EV. Bull Exp Biol (Moscow, U.S.S.R.) 1986; 2: 174-177

The antiepileptic effects of enomelanin, a natural oligomer obtained from pressing grapes, were analysed in animal models of focal and generalised tonic-clonic seizure activity. Electrocorticographic and motor manifestations were recorded. Enomelanin in these models was shown to possess a marked anticonvulsant property. 